\title{
Intraoperative Imaging Techniques to Visualize Hepatic (Micro)Perfusion: An Overview
}

\author{
Zühre Uz ${ }^{a, b}$ Lucinda Shen ${ }^{b, c}$ Dan M.J. Milstein ${ }^{d}$ Krijn P. van Lienden ${ }^{e}$ \\ Rutger-Jan Swijnenburga ${ }^{a}$ Can Ince $^{b, c}$ Thomas M. van Gulik ${ }^{a}$ \\ aDepartment of Surgery, Amsterdam UMC, University of Amsterdam, Amsterdam, \\ The Netherlands; ${ }^{b}$ Department of Translational Physiology, Amsterdam UMC, University \\ of Amsterdam, Amsterdam, The Netherlands; ' ${ }^{2}$ epartment of Intensive Care Adults, \\ Erasmus MC, Rotterdam, The Netherlands; ${ }^{d}$ Department of Oral and Maxillofacial Surgery, \\ Amsterdam UMC, University of Amsterdam, Amsterdam, The Netherlands; ${ }^{\text {e Department of }}$ \\ Radiology, Amsterdam UMC, University of Amsterdam, Amsterdam, The Netherlands
}

\section{Keywords}

Hepatic microcirculation · Perfusion · Imaging techniques · Intraoperative perfusion imaging

\begin{abstract}
The microcirculation plays a crucial role in the distribution of perfusion to organs. Studies have shown that microcirculatory dysfunction is an independent predictor of morbidity and mortality. Hence, assessment of liver perfusion offers valuable information on the (patho)physiological state of the liver. The current review explores techniques in perfusion imaging that can be used intraoperatively. Available modalities include dynamic contrast-enhanced ultrasound, handheld vital microscopes, indocyanine green fluorescence angiography, and laser contrast speckle imaging. Dynamic contrast-enhanced ultrasound relays information on deep tissue perfusion and is a commonly used technique to assess tumor perfusion. Handheld vital microscopes provide direct visualization of the sinusoidal architectural structure of the liver, which is a unique feature of this technique. Intraoperative fluorescence imaging uses indocyanine green, a dye that is administered intravenously to visualize microvascular perfusion when excited using near-infrared light. Laser speckle contrast imaging produces non-contact large surface-based tissue perfusion imaging free from movement- or pressure-related artefacts. In this review, we discuss the intrinsic advantages and disadvantages of these techniques and their clinical and/or scientific applications.

(c) 2020 The Author(s)

Published by S. Karger AG, Basel
\end{abstract}

Zühre Uz and Lucinda Shen contributed equally to this work. 


\section{Introduction}

The microcirculation sustains perfusion and delivery of oxygen to metabolically active tissues, and thus should be considered central to (patho)physiology. Unfortunately, considering the importance of organ perfusion, robust and detailed studies on hepatic microcirculation are lacking. Systemic hemodynamic parameters alongside laboratory results are examined perioperatively. However, many published papers reveal an incoherence between the macrocirculation and the microcirculation as central to pathophysiology, especially within septic patients $[1,2]$. Research has determined that a significant correlation between impaired microcirculatory flow and poor patient outcome exists despite stable hemodynamics suggesting that early assessment and correction of the microcirculation within critically ill patients would significantly reduce mortality and morbidity rates [3].

Alterations in hepatic microcirculation can be explained as the result of multiple causes that are of intraoperative interest. Hepatic pathological conditions can be characterized by regional and overall changes in perfusion. For example, during liver transplant surgery, the donor liver is (repeatedly) subjected to ischemia reperfusion injury. Poor hepatic arterial and venous blood flow into the transplant liver raises the risk of acute graft rejection (AGR) [4]. The ability to predict patients at risk of AGR, through perfusion monitoring, enables early interventions to treat and thereby reduce morbidity and mortality rates.

Perfusion imaging techniques provide real-time quantitative and qualitative measurements of flow dynamics, angioarchitecture, and morphology of the hepatic microcirculation. The aim of this review is to describe the variety of technologies used for intraoperative imaging of liver perfusion in humans and to examine their strengths, weaknesses, and clinical applications. Within these techniques, four domains are addressed: (1) dynamic contrastenhanced ultrasound (DCE-US), (2) handheld vital microscopy (HVM), (3) indocyanine green (ICG) fluorescence imaging, and (4) near-infrared laser speckle contrast imaging (LSCI).

\section{Dynamic Contrast-Enhanced Ultrasound}

For DCE-US, a contrast agent (CA) composed of millions of microbubbles has to be injected intravenously. A transducer is placed within the region of interest (ROI), which will emit a high-frequency soundwave. These soundwaves will collide with the surrounding tissues and reflect back, therefore producing a real-time image of the ROI [5]. The injected microbubbles have a higher degree of echogenicity when compared to its surrounding tissue, thereby creating a sonogram with a markedly increased contrast.

The gas-filled microbubbles are approximately 1-10 $\mu \mathrm{m}$ in diameter, making them true blood pool agents. This enables direct observation of the microcirculation and hemodynamic quantifications with exquisite spatial resolution [6]. Injection of the CA yields linear data production, which can generate time intensity curves (TICs). Three functions can be calculated using the TIC: (1) peak intensity, (2) area under the curve, and (3) wash-in phase [7]. The three mentioned functions calculated using the TIC provide information related to the fractional liver blood volume. Also, when the time to peak intensity and the slope of the wash-in is calculated, it provides information related to liver blood flow. In summary, these calculations from the TICs provide information on the fractional blood volume and blood flow in the liver, which are important parameters for liver perfusion and indirectly, for liver function. For example, the area under the curve and area under the wash-out have been shown experimentally by Gauthier et al. [8] to be reliable in assessing liver tumor perfusion.

There are currently three common DCE-US CA used worldwide. SonoVue ${ }^{\circledR}$ (Bracco SpA, Milan, Italy) is the most commonly adopted CA in Europe and recently, has also been approved 
for hepatic perfusion imaging in adults and pediatric patients by the FDA in $2016[9,10]$. Definity ${ }^{\circledR} /$ Luminity ${ }^{\circledR}$ (Lantheus Medical, Billerica, MA, USA) is commonly used in the US but has also recently been licensed in Canada and Australia $[9,10]$. Sonazoid ${ }^{\mathrm{TM}}$ (Daiichi-Sankyo, GE, Tokyo, Japan) is mostly used in Japan and South Korea $[9,10]$. It contains a hydrogenated egg phosphatidylserine on its outer shell which is phagocytosed by Kupffer cells of the liver while maintaining its echogenicity; this not only enables observation of hepatic perfusion but also the Kupffer cells [10].

\section{Clinical Applications}

The use of DCE-US in hepatic surgery was first described in 2006 by Leen et al. [11]; they discovered that DCE-US was more sensitive in detecting liver metastasis when compared to contrast-enhanced CT or MRI. In 2013, a consensus was published describing the use of DCE-US in the characterization of focal liver lesions (FLLs) [12]. The consensus states that benign FLL will show peripheral nodular enhancement during the arterial phase and incomplete filling during the late phase. Malignant FLLs seem to be less well defined; however, some have argued that arterial phase hypervascularity seem to be the most reliable characteristic.

In surgery, DCE-US is used to guide resection margins and detect additional tumor lesions [13]. In a study by Jung et al. [14], the use of DCE-US with a bolus injection of SonoVue CA in combination with a high-resolution linear probe (of up to $15 \mathrm{MHz}$ ) detected additional tumor lesions previously missed by contrast-enhanced CT or MRI. In the study, which consisted of a cohort of 20 patients, additional tumors (with a diameter of 4-19 $\mathrm{mm}$ ) were seen in 9 of these patients using DCE-US and confirmed by pathology [14]. The ability to detect small tumor lesions allows complete resection in surgery for liver metastases. Intraoperative DCE-US contributed towards modifying surgical management in $30 \%$ of cases [15].

As CAs are blood pool agents, this enables better characterization of FLL during the portal and late venous phase. Additionally, the CAs Levovist and Sonazoid are phagocytosed by Kupffer cells, therefore prolonging the resonating period. This enhances stability of the CA enabling whole liver scanning (which Jung et al. [14] have reported to take $\sim 10 \mathrm{~min}$ ) and also helps in differentiating between benign and malignant tumors [16].

\section{Advantages and Disadvantages}

The main advantages of DCE-US are derived from the characteristics of the CAs. Studies have shown that the gas-filled microbubbles have very low incidents of cardio-, hepatic, and nephrotoxicity [16]. In combination with the non-ionizing nature of DCE-US, this makes the current modality most suitable for pediatrics and critically ill patients. The strict blood pool confinement of the CAs allows better characterization of FLLs and detection of smaller lesions in the liver $[10,12]$.

However, the correct diagnosis using DCE-US requires extensive training and observation on behalf of the observer. It has been suggested that a learning curve is required with supervision by an expert in the field [10]. Since even millimeter deviations of the transducer on the liver surface will have effects on blood flow measurements. Furthermore DCE-US is not recommended for patients with chronic heart failure and/or fatty livers. Compromised back flow of the blood from the right atrium during chronic heart failure may cause disruptions in measurement of the arterial phase, and fatty liver reduced the sensitivity and specificity of DCE-US [7, 10]. It should also be taken into consideration that different CAs will have different hepatic transit times. Studies have shown that Lenovist has a significantly longer transit time when compared to SonoVue and Luminity, and thus results should be interpreted with care [17].

Limited by the surface area of the transducer, DCE-US imaging only allows the evaluation of one FLL with one bolus injection of the CA. Therefore, the evaluation of more than one FLL will require multiple injections of the CA [17].

\section{Karger's}


Fig. 1. Examples of liver perfusion imaging by handheld vital microscopy and indocyanine green (ICG) fluorescence imaging. a Screenshot of human hepatic microcirculation obtained using the Cytocam Handheld Video Microscope Imaging System (or IDFI). RBCs can be observed individually, the sinusoids (S) are easily imaged and also larger vessels (LV) branching out of the portal field (PF). b Real-time image of liver perfusion after ICG (green fluorescence) injection using an intraoperative fluorescence camera. Following clamping of the left portal vein and left hepatic artery, clear demarcation of liver perfusion is visualized, which guides the surgeon during performing, in this case, a left hemihepatectomy.
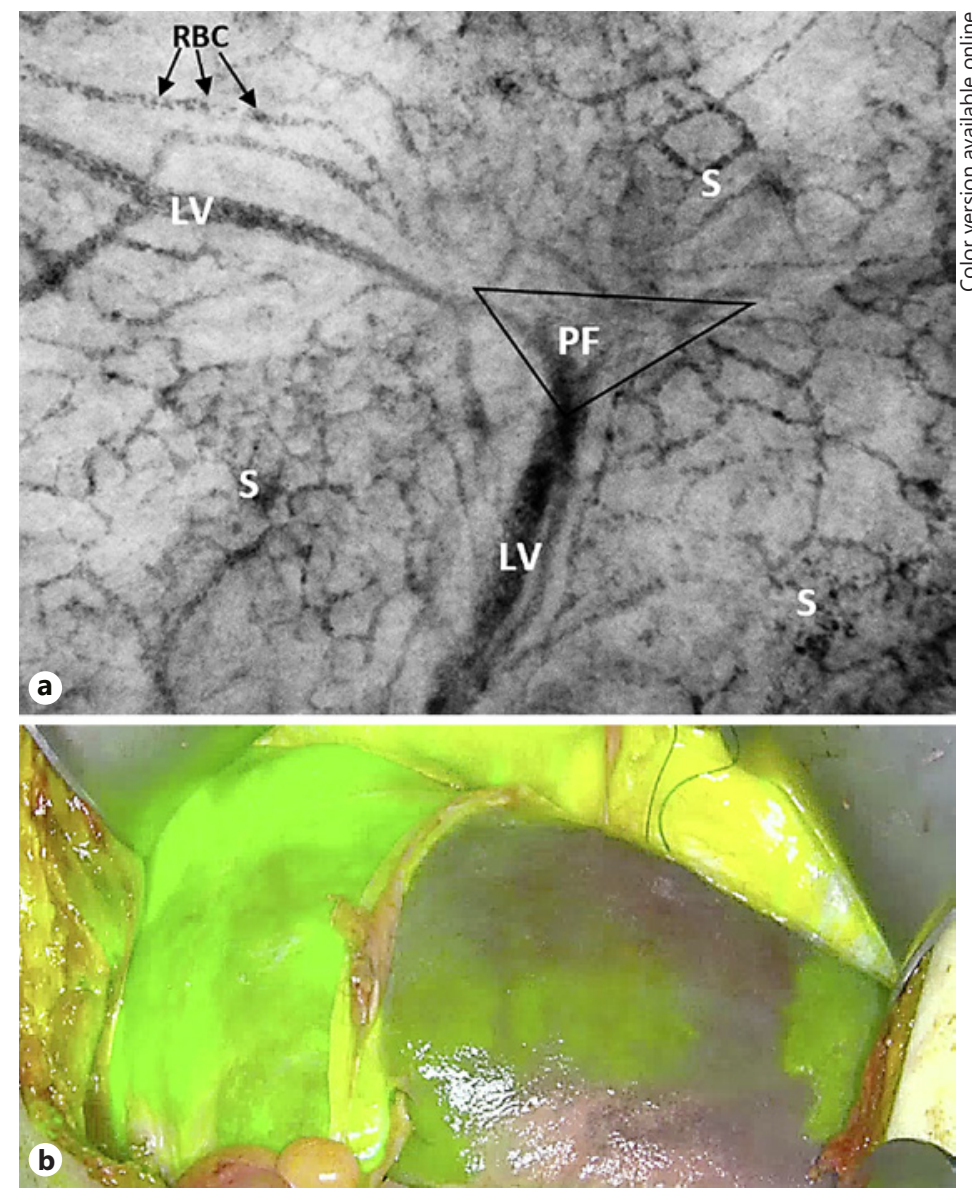

\section{Handheld Vital Microscopy}

There are currently three existing types of HVM: (1) orthogonal polarization spectral imaging (OPSI) - first generation, (2) sidestream dark-field imaging (SDFI) - second generation, and (3) incident dark-field imaging (IDFI) - third generation, which is the newest HVM. The use of OPSI was first documented by Groner et al. [18], who investigated characteristic changes in nailfold capillaries for the diagnosis and treatment of hematological disorders. Briefly, OPSI operates by illuminating the ROI with polarized light at $548 \mathrm{~nm}$. Light waves capable of penetrating deeper into the tissue will scatter and depolarize, while waves reflected back from the superficial surface will remain polarized. The reflected waves pass through an orthogonally placed polarizer lens to filter out surface reflection before being collected and recorded by the charge-coupled device camera producing a real-time image. Hemoglobin is able to absorb light at $548 \mathrm{~nm}$, therefore appearing dark on the generated image. This grants direct visualization of the RBC containing hemoglobin within the microvascular lumen, hence the microcirculation [18].

Observation of the complete microcirculatory network opened up a new field of microcirculation research using HVM. However, OPSI is highly sensitive to the backscatter of light, which leads to blurring [19]. This led to the development of the second-generation SDFI. Based on the same principles, SDFI uses concentrically placed LED lights around the probe tip, expelling greenlights with a wavelength of $530 \mathrm{~nm}$ (within the hemoglobin light absorption range). This reduced surface reflection generates images with a superior contrast between 
the microcirculation and background tissue. SDFI was validated against OPSI as reported by Goedhart et al. [20] on the nailfold and in the sublingual cave microcirculation.

The third and most current generation of HVM uses IDFI (Fig. 1a). IDFI was first described by Sherman et al. [21] in 1971. Adaptations in the concept of epi-illuminating the organ surface for the study of microcirculation has led to the development of the CytoCam (Braedius Medical, Huizen, The Netherlands) device. CytoCam has concentrically placed, high-brightness LED lights, a factor 4 optical magnification system, and a larger imaged area. This provided $30 \%$ more visualization of the microcirculatory network and superior image resolution when compared to SDFI or OPSI [22]. Furthermore, the LED lights have a shorter illumination pulse time, further reducing blurring of the RBCs.

Tissue perfusion depends on the density, distribution, and diameters of the capillaries in combination with blood viscosity and driving pressure across the capillaries. The two main hemodynamic principles governing oxygen supply to tissue are convection and diffusion. Convection is quantified by flow, and diffusion is quantified by the density of the perfused (and thus functional) microvessels. Within IDFI, flow is calculated by scoring the predominant type of flow across microvessels. This generates the microvascular flow index, a semiquantitative score ranging from 0 (no flow) to 3 (continuous flow). To quantify diffusion, the proportion of perfused vessels (PPV), the perfused vessel density (PVD), and total vessel density (TVD) are determined. PPV is calculated by scoring all vessels with a flow score greater than 1. PVD is calculated by multiplying TVD by PPV. Both PVD and TVD are expressed in $\mathrm{mm} / \mathrm{mm}^{2}$. Each score is determined for small microvessels with a cut-off diameter of 25 $\mu \mathrm{m}[23]$.

\section{Clinical Applications}

Clinical introduction of OPSI in the liver was first documented by Puhl et al. [24] in 2003. For the first time, the architectural structure of the human hepatic sinusoidal circulation could be directly observed using a noninvasive approach. OPSI aided quantitative analysis of hepatic microcirculation, which provided significant insight into hepatic physiology. Since then, OPSI was employed mostly for investigating liver graft dysfunction. In particular, it was used to monitor changes within the hepatic microcirculation before and after liver transplantation for predictive signs of graft rejection [25]. This has great clinical significance enabling surgeons to identify patients at risk of AGR during surgery. SDFI has been used clinically to evaluate changes within the hepatic microcirculation following major or minor liver resection [26]. Changes were measured in RBC velocity, sinusoidal diameter, and functional sinusoidal density between damaged and undamaged liver parenchyma. A weak correlation was identified between an increase in RBC velocity and volume of liver resected. To date, the only paper published on the use of IDFI in the liver is by Uz et al. [27], who measured liver microcirculation in 18 patients undergoing major hepatectomy with and without preoperative unilateral portal vein embolization. They found a significantly higher hepatic microvascular density in the non-embolized lobe when compared to the embolized lobe, as the result of angiogenesis occurring in the hypertrophic, non-embolized lobe after portal vein embolization [27].

\section{Advantages and Disadvantages}

The major advantage of HVM when compared to the other techniques reviewed in this paper is the ability to produce dynamic structural images of the microcirculation. The lightweight and portable nature of HVM makes it very practical and suitable for inspection of the microcirculation in the operating room [28]. Additionally, HVM promotes visualization of leukocyte kinetics in the microcirculation using space-time diagrams, a parameter generated by the semiautomated analysis software AVA 3.0 (MicroVision Medical Ltd, Amsterdam, The

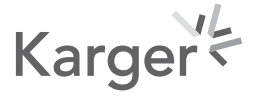


Netherlands). Monitoring of intraoperative inflammatory status of the hepatic microcirculation can provide pivotal information on the local interaction of leukocytes, endothelial cells, and microcirculatory flow [29].

HVM imaging is, however, not without limitations. Measurements are highly subjective to movement and pressure artefacts. Induced pressure inhibits the flow of RBCs through the microvessels rendering these vessels invisible in the field of view [23]. Therefore, a learning curve must be undertaken by the investigator with an expert in the field. Furthermore, the surface area of the microscopes limited the field of view to approximately $7,783 \mu \mathrm{m}^{2}$. Therefore, information of whole liver perfusion will require scanning of the liver surface. With a maximum of $500 \mu \mathrm{m}$ focus depth, only surface perfusion information can be gathered from HVM, although in most pathological conditions, this is sufficient to focus through the liver capsule and capture the sinusoidal microcirculation [27].

\section{Indocyanine Green Fluorescence Imaging}

ICG fluorescence imaging is a real-time imaging technique to enhance the visualization of anatomical structures and give detailed functional information of the vascular network during surgery. The principles are simple: ICG is a molecule with a mass of 776 Da that becomes almost exclusively albumin bound following intravenous injection [30]. The removal of ICG from the blood is strictly through the uptake by hepatocytes and excreted via bile after $8 \mathrm{~min}$ of injection. ICG fluoresces at the near-infrared wavelength $(790-805 \mathrm{~nm})$, which is invisible to the human eye, and detection requires the utility of specialized near-infrared cameras. In recent years, different near-infrared fluorescence camera systems have been developed for both open and minimally invasive surgery. The dye has no adverse effects, at the standard clinical dosage of $0.1-0.5 \mathrm{mg} / \mathrm{mL} / \mathrm{kg}$ [31].

\section{Clinical Applications}

The use of ICG imaging for intraoperative liver perfusion assessment can be broadly categorized into (1) liver mapping (see Fig. 1b), (2) tumor visualization, and (3) liver graft perfusion evaluation [32]. It is also the only technique described by this review which can be applied laparoscopically. During laparoscopic surgery, liver palpation and gross inspection by the surgeon are limited. ICG can aid in further detection of superficial nodules, marking resection margins, and identifying liver intersegmental boundaries [33]. Laparoscopic surgeries used PINPOINT near-infrared imaging system to generate overlaid images of near-infrared and visible light so that the color difference between segmental boundaries are distinguishable $[34,35]$. The color difference between the pseudocolor generated by PINPOINT near-infrared imaging and the red-purple parenchyma allows the identification of segmental boundaries with and without perfusion, thus guiding surgeons during laparoscopic surgery [33].

Since ICG is strictly removed by the liver, the ICG clearance test has been used for the assessment of liver function [36]. In actual fact, it is the most widely applied quantitative liver function test in the selection of patients considered for major liver resection, as exemplified by the LiMON technology. Following intravenous administration of the ICG dye, the concentration of the dye will fall exponentially. Measurements of ICU clearance are performed in serial blood samples but can also be determined noninvasively, through pulse dye densitometry using an optical sensor placed at the fingertip [37,38]. The use of this technology was found to be the best discriminating preoperative test for evaluating hepatic functional reserve in patients diagnosed with HCC [36].

The characteristic of ICG fluorescence can aid in the differentiation of hepatic lesions. For example, in the case of metastasis, the dye is retained in the stromal tissue within the tran- 
sition region between the parenchyma and the tumor, whereas in hepatocellular carcinoma, the fluorescence is observed inside the tumor [38]. Regardless, guidelines are still yet not available for ICG fluorescence-directed differential diagnosis of malignant and benign tumors, and thus, data should be interpreted with care.

The use of ICG fluorescence imaging in liver transplant surgery aids in predicting AGR through the assessment of (1) bile duct vascularization and biliary anastomosis, (2) perfusion evaluation of the arterial and portal anastomotic sites, and (3) fluorescence intensity [31,37]. The outcome of these studies highly encouraged the use of ICG imaging during liver transplantation, providing safe objectifiable perfusion information during organ transplantations and allowing real-time modification of surgical techniques, thereby reducing graft dysfunction $[31,37]$.

\section{Advantages and Disadvantages}

The ICG technique is fairly straightforward and does not require an extensive learning curve, unlike the other modalities discussed in the current review (i.e., HVM and DCE-US). ICG imaging allows for the easy visualization of segmental boundaries, which provides important anatomical information for resection planning and is also the only modality discussed which is applicable to laparoscopic surgeries. The ICG dye can also be injected into the human vascular system with virtually no side effects.

Criticism of its limitations to the lack of quantitative data generated by ICG fluorescence imaging is a disadvantage of this modality. Only visual information is available; therefore, interpretation is up to the surgeons' experience with using ICG. Without absolute values, this makes interpretation and comparison of perfusion parameters difficult. Furthermore, limited by the ability for near-infrared light to penetrate deep into the tissue, only superficial lesions can be visualized. Moreover, intraoperative ultrasonography is also usually adopted to improve ICG imaging accuracy. Lastly, ICG contamination spillage via lymphatic leakage can obscure the surgeon's field of view [32]. The leaked ICG will bind to proteins within the abdominal cavity and emits a strong fluorescent signal which can obstruct critical structures.

\section{Laser Speckle Contrast Imaging}

With LSCI, a near-infrared laser light is used to illuminate the tissue surface, which will reflect light back as speckles. Constant change in the contour of the tissue surface will create irregular backscatter of speckles. A measurement camera will record the speckles as constructive and destructive interference patterns (light and dark speckles). The flow of RBCs underneath the tissue surface will cause a dynamic change within the interference pattern, which is recorded as blurring. Thus, increased blood flow will correlate with an increased blurring. The level of blurring can be quantified by measuring the spatial contrast of the intensity variations, representing the average velocity and concentration of moving RBCs. LSCI generates a 2-dimensional blood flow map of the organ at a single given time point, permitting the investigator to identify areas of high or low perfusion $[39,40]$.

\section{Clinical Applications}

Currently, the only published clinical trial carried out using LSCI in investigating the liver microcirculation was during liver resections in 10 patients in a pilot study [39]. Analyzable images of the hepatic microcirculation were successfully measured in all patients using LSCI; the total time spent making the measurement and conducting the analysis of each image was 5 min [39].

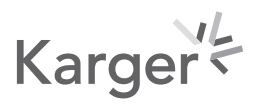




\section{Advantages and Disadvantages}

LSCI has many advantages: it produces whole organ perfusion maps almost instantaneously, rendering LSCI suitable for dynamic microcirculatory functional tests. It is low-cost to run and yields highly reproducible results. Most importantly, it does not come into contact with the organ surface and therefore does not suffer from pressure artefacts associated with tissue contact (unlike HVM).

However, as the technique functions on reading the backscatter of light to determine perfusion, any movement within the tissue surface is registered as perfusion and therefore, LSCI is sensitive to movement artefacts. To determine the degree of faulty measurements associated with movement of the target tissue, Mahe et al. [41] used an opaque patch, placed on the ROI as a reference point for movement induced by the skin. The difference measured was not significant in minute movements; although these movements slightly interfere with the measurements, the measurements remain largely comparable between tiny shifts in space. Furthermore, values which are generated by LSCI are expressed as a mean of RBC velocity and concentration and therefore do not give absolute data but rather represent an estimation or relative measurement [42]. The depth covered by LSCI is superficial, as light can only penetrate for approximately 300 $\mu \mathrm{m}$ into the tissue, thus lacking information on perfusion deeper in the target organ.

\section{Discussion}

In this review, four modalities of measuring liver perfusion intraoperatively were described with respect to their advantages, disadvantages, and clinical applications (Table 1). The choice of technique used is highly dependent on the research or clinical question.

For measurements concerning deeper liver perfusion, DCE-US has the greatest depth of focus when compared to HVM, ICG fluorescence imaging, and LSCI. This gives DCE-US greater application in deep tissue perfusion imaging, whereas HVM and LSCI can only yield information from subsurface (superficial) tumor lesions. Deep tissue perfusion imaging combined with the ability to detect additional tumor lesions have resulted in alterations to surgical management strategies using DCE-US. However, operation of DCE-US (in addition with ICG fluorescence imaging) requires the injection of a CAs. Although these CAs have shown low incidents of toxicity, under situations where intravenous injections are not possible, the employment of HVM and LSCI can be used.

All modalities discussed in the current review are applicable to open laparotomic surgeries, which are considered the standard for liver surgeries; however, ICG fluorescence imaging is the only modality which can also be applied laparoscopically. The laparoscopic approach has been less favored for the lack of tactile sensation and gross inspection of the liver [43]. ICG fluorescence imaging using PINPOINT near-infrared imaging provides a realtime overlay of fluorescence and white-light imaging, without disturbance of the surgical field. It can give the surgeon navigation information for both surface lesions and for anatomical segmental perfusion to guide surgeons for safer liver resections [33]. Not only does laparoscopic surgery have better cosmetic effects, it also has superior results for total blood loss rates, transfusion rates, and recovery times [43].

Currently, only ICG fluorescence imaging and DCE-US have been implemented for clinical use; however, HVM has undergone a number of technological developments with the aim of introducing these devices for routine clinical practice. HVM allows the direct visualization of RBCs, which should be considered the golden standard for assessing perfusion [1]. This direct observation of the microcirculatory angioarchitecture yields quantitative information on diffusion, convection, and inflammatory status of the microcirculation, which can be used for monitoring the patient's hemodynamic status at the bedside or in research. 


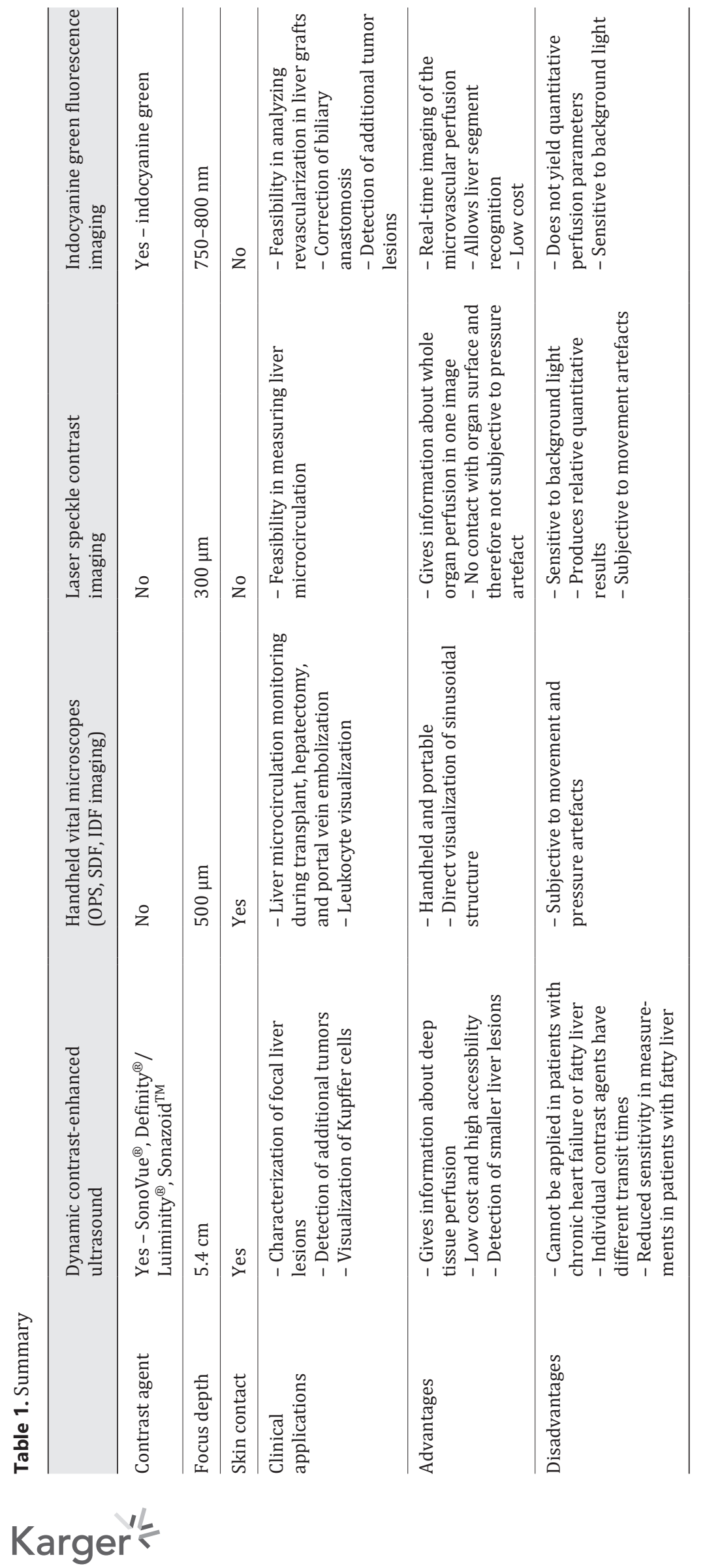


This review is not without limitations. Firstly, laser Doppler was not discussed in detail as a potential technique to assess intraoperative hepatic perfusion [44]. Laser Doppler flowmetry presents tissue perfusion as a single value at one point rather than an image of tissue perfusion. We therefore believe that this modality did not fit in the scope of the current article. Secondly, due to the narrative style of the review, technical variations of the discussed imaging modality were possibly not examined in detail. The aim of this review was to give readers a general overview of widely available techniques for intraoperative perfusion imaging. Thirdly, this review only focused on approaches for intraoperative perfusion imaging rather than for the entire perioperative period. Therefore, application of dynamic contrast-enhanced CT and MRI for this purpose were not considered in this review.

In conclusion, the different modalities described in this review all possess intrinsic advantages and disadvantages. The choice of the technique used should depend on the output data of interest. DCE-US gives information on deep tissue perfusion, whereas HVM, ICG, and LSCI are only superficially applicable. DCE-US has found clinical use, whereas HVM, ICG, and LSCI are more research-focused instruments. However, DCE-US and HVM require contact with the organ surface and are therefore susceptible to pressure artefacts, whereas LSCI and ICG are sensitive to light scatter. Regardless of choice, the challenge remains in the interpretation of the quantitative outputs acquired and their clinical significance.

\section{Conflict of Interest Statement}

Prof. Dr. Ince is listed as one of the inventors of the SDFI on related patents commercialized by Microvision Medical (MVM) under a license from the Amsterdam UMC. He holds no shares of the company and receives no royalties or benefits from this license. He has not been involved with MVM for more than 6 years. Cytocam-IDFI was developed by the company Braedius Medical (BM), which is owned by a relative of Prof. Dr. Ince; however, he has no financial ties with BM of any sort (i.e., never owned shares or received consultancy or speaker fees). Prof. Dr. Ince runs an internet site which offers services (e.g., training, courses, analysis) related to clinical microcirculation (https://microcirculationacademy.org). The other authors declare that they have no competing interests.

\section{Funding Sources}

The review is funded through internal department funds.

\section{Author Contributions}

L.S. and Z.U. contributed to the initial literature search for the content of the manuscript and design of the manuscript. D.M.J.M. and K.P.v.L. contributed significantly to the drafting of the manuscript and gave critical review of the content. R.-J.S. provided materials needed for the making of Figure 1. C.I. contributed to the drafting of the manuscript, gave his critical feedback of the content, and was involved in the design of the manuscript and provided materials needed for the making of Figure 1. T.M.v.G. gave critical feedback of the manuscript content, was involved in the design of the manuscript, and provided materials for making Figure 1. All authors contributed to the drafting of the final manuscript. 


\section{References}

1 Ince C. Hemodynamic coherence and the rationale for monitoring the microcirculation. Crit Care. 2015;19(S3 Suppl 3):S8.

2 Hernandez G, Regueira T, Bruhn A, Castro R, Rovegno M, Fuentealba A, et al. Relationship of systemic, hepatosplanchnic, and microcirculatory perfusion parameters with 6-hour lactate clearance in hyperdynamic septic shock patients: an acute, clinical-physiological, pilot study. Ann Intensive Care. 2012 Oct;2(1):44.

3 Edul VS, Enrico C, Laviolle B, Vazquez AR, Ince C, Dubin A. Quantitative assessment of the microcirculation in healthy volunteers and in patients with septic shock. Crit Care Med. 2012 May;40(5):1443-8.

4 Pulitano C, Joseph D, Sandroussi C, Verran D, Ho P, Debiasio A, et al. Postreperfusion microcirculatory derangements after liver transplantation: relationship to hemodynamics, serum mediators, and outcome. Liver Transpl. 2017 Apr;23(4):527-36.

5 Coatney RW. Ultrasound imaging: principles and applications in rodent research. ILAR J. 2001;42(3):233-47.

6 Wilson SR, Burns PN. Microbubble-enhanced US in body imaging: what role? Radiology. 2010 Oct;257(1): 24-39.

7 Fröhlich E, Muller R, Cui XW, Schreiber-Dietrich D, Dietrich CF. Dynamic contrast-enhanced ultrasound for quantification of tissue perfusion. J Ultrasound Med. 2015 Feb;34(2):179-96.

8 Gauthier M, Leguerney I, Thalmensi J, Chebil M, Parisot S, Peronneau P, et al. Estimation of intra-operator variability in perfusion parameter measurements using DCE-US. World J Radiol. 2011 Mar;3(3):70-81.

9 Pang EH, Chan A, Ho SG, Harris AC. Contrast-Enhanced Ultrasound of the Liver: Optimizing Technique and Clinical Applications. AJR Am J Roentgenol. 2018 Feb;210(2):320-32.

10 Claudon M, Dietrich CF, Choi BI, Cosgrove DO, Kudo M, Nolsøe CP, et al. Guidelines and Good Clinical Practice Recommendations for Contrast Enhanced Ultrasound (CEUS) in the Liver - Update 2012: A WFUMB-EFSUMB Initiative in Cooperation with Representatives of AFSUMB, AIUM, ASUM, FLAUS and ICUS. Ultrasound Med Biol. 2013 Feb;39(2):187-210.

11 Leen E, Ceccotti P, Moug SJ, Glen P, MacQuarrie J, Angerson WJ, et al. Potential value of contrast-enhanced intraoperative ultrasonography during partial hepatectomy for metastases: an essential investigation before resection? Ann Surg. 2006 Feb;243(2):236-40.

12 Jang JY, Kim MY, Jeong SW, Kim TY, Kim SU, Lee SH, et al. Current consensus and guidelines of contrast enhanced ultrasound for the characterization of focal liver lesions. Clin Mol Hepatol. 2013 Mar;19(1):1-16.

13 Torzilli G, Botea F, Procopio F, Donadon M, Balzarini L, Lutman F, et al. Use of contrast-enhanced intraoperative ultrasonography during liver surgery for colorectal cancer liver metastases - Its impact on operative outcome. Analysis of a prospective cohort study. Eur J Cancer, Suppl. 2008;6(11):16-23.

14 Jung EM, Clevert DA, Schreyer AG, Schmitt S, Rennert J, Kubale R, et al. Evaluation of quantitative contrast harmonic imaging to assess malignancy of liver tumors: a prospective controlled two-center study. World J Gastroenterol. 2007 Dec;13(47):6356-64.

15 Sietses C, Meijerink MR, Meijer S, van den Tol MP. The impact of intraoperative ultrasonography on the surgical treatment of patients with colorectal liver metastases. Surg Endosc. 2010 Aug;24(8):1917-22.

16 Chiorean L, Tana C, Braden B, Caraiani C, Sparchez Z, Cui XW, et al. Advantages and Limitations of Focal Liver Lesion Assessment with Ultrasound Contrast Agents: Comments on the European Federation of Societies for Ultrasound in Medicine and Biology (EFSUMB) Guidelines. Med Princ Pract. 2016;25(5):399-407.

17 Haendl T, Strobel D, Neureiter D, Esser K, Frieser M, Hahn EG, et al. [A comparative study of the hepatic transit time (HTT) of different ultrasound contrast agents in patients with liver metastases and healthy controls]. Ultraschall Med. 2010 Dec;31(6):582-8.

18 Groner W, Winkelman JW, Harris AG, Ince C, Bouma GJ, Messmer K, et al. Orthogonal polarization spectral imaging: a new method for study of the microcirculation. Nat Med. 1999 Oct;5(10):1209-12.

19 Treu CM, Lupi O, Bottino DA, Bouskela E. Sidestream dark field imaging: the evolution of real-time visualization of cutaneous microcirculation and its potential application in dermatology. Arch Dermatol Res. 2011 Mar;303(2):69-78.

20 Goedhart PT, Khalilzada M, Bezemer R, Merza J, Ince C. Sidestream Dark Field (SDF) imaging: a novel stroboscopic LED ring-based imaging modality for clinical assessment of the microcirculation. Opt Express. 2007 Nov; 15(23):15101-14.

21 Sherman H, Klausner S, Cook WA. Incident dark-field illumination: a new method for microcirculatory study. Angiology. 1971 May;22(5):295-303.

22 Gilbert-Kawai E, Coppel J, Bountziouka V, Ince C, Martin D, Ahuja V, et al.; Caudwell Xtreme Everest and Xtreme Everest 2 Research Groups. A comparison of the quality of image acquisition between the incident dark field and sidestream dark field video-microscopes. BMC Med Imaging. 2016 Jan;16(1):10.

23 Ince C, Boerma EC, Cecconi M, De Backer D, Shapiro NI, Duranteau J, et al.; Cardiovascular Dynamics Section of the ESICM. Second consensus on the assessment of sublingual microcirculation in critically ill patients: results from a task force of the European Society of Intensive Care Medicine. Intensive Care Med. 2018 Mar; 44(3):281-99.

24 Puhl G, Schaser KD, Vollmar B, Menger MD, Settmacher U. Noninvasive in vivo analysis of the human hepatic microcirculation using orthogonal polorization spectral imaging. Transplantation. 2003 Mar;75(6):756-61.

25 Puhl G, Schaser KD, Pust D, Köhler K, Vollmar B, Menger MD, et al. Initial hepatic microcirculation correlates with early graft function in human orthotopic liver transplantation. Liver Transpl. 2005 May;11(5):555-63. 
26 Nilsson J, Eriksson S, Blind PJ, Rissler P, Sturesson C. Microcirculation changes during liver resection - a clinical study. Microvasc Res. 2014 Jul; 94:47-51.

27 Uz Z, Ince C, Rassam F, Ergin B, van Lienden KP, van Gulik TM. Assessment of hepatic microvascular flow and density in patients undergoing preoperative portal vein embolization. HPB (Oxford). 2019;21(2):187-94.

28 Bezemer R, Bartels SA, Bakker J, Ince C. Clinical review: clinical imaging of the sublingual microcirculation in the critically ill - where do we stand? Crit Care. 2012 Jun;16(3):224.

29 Zühre U, Gulik TM, Aydemirli MD, Guerci P, Ince Y, Ergin B, et al. Identification and quantification of human microcirculatory leukocytes using handheld video microscopes at the bedside. J Appl Physiol (1985). 2018; 124(6):1550-7.

30 Yoneya S, Saito T, Komatsu Y, Koyama I, Takahashi K, Duvoll-Young J. Binding properties of indocyanine green in human blood. Invest Ophthalmol Vis Sci. 1998 Jun;39(7):1286-90.

31 Panaro F, Benedetti E, Pineton de Chambrun G, Habibeh H, Leon P, Bouyabrine H, et al. Indocyanine green fluorescence angiography during liver and pancreas transplantation: a tool to integrate perfusion statement's evaluation. Hepatobiliary Surg Nutr. 2018 Jun;7(3):161-6.

32 Huang SW, Ou JJ, Wong HP. The use of indocyanine green imaging technique in patient with hepatocellular carcinoma. Transl Gastroenterol Hepatol. 2018 Nov;3:95. Available from: http://tgh.amegroups.com/article/ view/4709.

33 Terasawa M, Ishizawa T, Mise Y, Inoue Y, Ito H, Takahashi Y, et al. Applications of fusion-fluorescence imaging using indocyanine green in laparoscopic hepatectomy. Surg Endosc. 2017 Dec;31(12):5111-8.

34 Ueno M, Hayami S, Sonomura T, Kawai M, Hirono S, Okada KI, et al. Concomitant Use of Indocyanine Green Fluorescence Imaging and Interventional Radiology for Detection of Liver Segments During Laparoscopic Anatomical Liver Resection: Pilot Feasibility Study. Surg Laparosc Endosc Percutan Tech. 2019 Aug;29(4): 242-6.

35 Cheung TT, Ma KW, She WH, Dai WC, Tsang SH, Chan AC, et al. Pure laparoscopic hepatectomy with augmented reality-assisted indocyanine green fluorescence versus open hepatectomy for hepatocellular carcinoma with liver cirrhosis: A propensity analysis at a single center. Asian J Endosc Surg. 2018 May;11(2):104-11.

36 Rassam F, Olth of PB, Bennink RJ, van Gulik TM. Current Modalities for the Assessment of Future Remnant Liver Function. Visc Med. 2017 Dec;33(6):442-8.

37 Figueroa R, Golse N, Alvarez FA, Ciacio O, Pittau G, Sa Cunha A, et al. Indocyanine green fluorescence imaging to evaluate graft perfusion during liver transplantation. HPB (Oxford). 2019 Apr;21(4):387-92.

38 Baiocchi GL, Diana M, Boni L. Indocyanine green-based fluorescence imaging in visceral and hepatobiliary and pancreatic surgery: state of the art and future directions. World J Gastroenterol. 2018 Jul;24(27):2921-30.

39 Eriksson S, Nilsson J, Lindell G, Sturesson C. Laser speckle contrast imaging for intraoperative assessment of liver microcirculation: a clinical pilot study. Med Devices (Auckl). 2014 Jul; 7:257-61.

40 Eriksson S, Nilsson J, Sturesson C. Non-invasive imaging of microcirculation: a technology review. Med Devices (Auckl). 2014 Dec; 7:445-52.

41 Mahe G, Abraham P, Le Faucheur A, Bruneau A, Humeau-Heurtier A, Durand S. Cutaneous microvascular functional assessment during exercise: a novel approach using laser speckle contrast imaging. Pflugers Arch. 2013 Apr;465(4):451-8.

42 Boas DA, Dunn AK. Laser speckle contrast imaging in biomedical optics. J Biomed Opt. 2010 Jan-Feb;15(1): 011109.

43 Chen J, Li H, Liu F, Li B, Wei Y. Surgical outcomes of laparoscopic versus open liver resection for hepatocellular carcinoma for various resection extent. Medicine (Baltimore). 2017 Mar;96(12):e6460.

44 Richter S, Sperling J, Kollmar O, Menger MD, Schilling MK; Chirurgische Arbeitsgemeinschaft OP-Technik und OP-Strukturen of the Deutsche Gesellschaft für Chirurgie. Laser Doppler flowmetry of hepatic microcirculation during Pringle's maneuver: determination of spatial and temporal liver tissue perfusion heterogeneity. Eur Surg Res. 2010;44(3-4):152-8. 